\title{
Intratympanic Gentamicin Versus Labyrinthectomy: Inner Ear Sensitivity to Gentamicin and Impact on the Contralateral Labyrinth
}

\author{
Georgios Kontorinis (i] $\cdot$ Hiteshkumar Tailor $\cdot$ Margaret Gaggini $\cdot$ John A. Crowther \\ Department of Otolaryngology-Head and Neck Surgery, Queen Elizabeth University Hospital, Glasgow, UK
}

Objectives. To investigate the impact of labyrinthectomy and intratympanic (IT) gentamicin injections on the contralateral labyrinth, we also assessed the response of each individual semicircular canal to each IT gentamicin application.

Methods. We performed a pilot observational study on tertiary, referral, academic settings. Thirteen patients with unilateral vestibular pathology were organized into two groups, group I (seven patients) receiving IT gentamicin and group II undergoing labyrinthectomy (six patients). All patients underwent six-canal video-head-impulse test in predetermined time intervals. Patients receiving gentamicin were additionally tested 3 to 5 days after every sequential injection, until all ipsilateral canals were ablated, to determine the order of response to gentamicin. We recorded the vestibular-ocular reflex gains and the presence of covert/overt saccades for each canal.

Results. The posttreatment ipsilateral gains were abnormal. No patient from the gentamicin group developed abnormal contralateral responses, while patients undergoing labyrinthectomy had abnormal contralateral responses from at least one canal, even several months posttreatment. Finally, the lateral semicircular canal was the first one to be affected by IT gentamicin followed by the posterior canal: the superior canal was ablated last.

Conclusion. In our study, labyrinthectomy had an impact on the responses recorded from the contralateral ear, while IT gentamicin ablated the ipsilateral labyrinthine function without affecting the contralateral responses, possibly because of a milder, more gradual impact. We also show for the first time the order that IT gentamicin application affects the semicircular canals, with the lateral being the first to be affected.

Keywords. Gentamicin; Head Impulse Test; Inner Ear; Semicircular Canals; Vestibular-Ocular Reflex

\section{INTRODUCTION}

Radical treatments are part of the management of refractory, disabling vestibular disorders, such as Meniere's disease that are resistant to more conservative measures [1-4]. Intratympanic (IT) gentamicin injections is a well-recognized method for managing patients with unilateral Meniere's disease [1-3], while labyrinthectomy, although more invasive, has also been used as definite

- Received December 20, 2018

Revised April 1, 2019

Accepted April 8, 2019

- Corresponding author: Georgios Kontorinis

Department of Otolaryngology-Head and Neck Surgery, Queen Elizabeth

University Hospital, 1345 Govan Rd, Glasgow G51 4TF, UK

Tel: +44-1413478028, Fax: +44-1413478028

E-mail: gkontorinis@gmail.com treatment $[3,4]$. Both treatment options can ablate the vestibular function; however, the IT gentamicin injections have the significant advantage of expected hearing preservation, particularly in patients with serviceable hearing [1-4].

Lately, a very few studies have looked into the precise impact of gentamicin on the injected ear using vestibular tests, including the relatively newly introduced six-canal video-head-impulse test (vHIT) [5-10]. The six-canal vHIT offers a fast, noninvasive measurement of the function of each individual semicircular canal $[9,10]$. Using vHIT, previous studies managed to show the impact of IT gentamicin injections on the semicircular canals, confirming its vestibulotoxicity, highlighting that one IT injection might not be enough to ablate the ipsilateral vestibular function [5,7-9]. When it comes to labyrinthectomy, studies assessing the vestibular function are missing, mostly because it is

Copyright @ 2019 by Korean Society of Otorhinolaryngology-Head and Neck Surgery.

This is an open-access article distributed under the terms of the Creative Commons Attribution Non-Commercial License (http://creativecommons.org/licenses/by-nc/4.0)

which permits unrestricted non-commercial use, distribution, and reproduction in any medium, provided the original work is properly cited. 
assumed that following the surgical intervention no function is preserved. Additionally, the impact of either chemical or surgical ablation on the vestibular response of the contralateral ear has barely been investigated [11,12]. As such interventions ablate function completely on the applied side, any impact on the only remaining functional ear can be detrimental.

There is a lack of studies assessing the result of IT gentamicin injections and/or labyrinthectomy to the contralateral, "healthy" ear. Given the potential clinical significance of any abnormalities caused in the remaining functional ear, we aimed at investigating the impact of both IT gentamicin injections and labyrinthectomy on the contralateral ear using six-canal vHIT; we also examined the order that gentamicin ablates the semicircular canals.

\section{MATERIALS AND METHODS}

\section{Basic settings}

A pilot, qualitative, observational study was carried out in a tertiary, referral, university center. Ethical approval was granted by the Institution Audit Board. Informed consent was obtained by all enrolled patients.

\section{Patient selection}

We enrolled patients with unilateral vestibular pathology without any response to conservative, nonablating methods and/or with a medical indication for radical treatment. Following detailed consultation and meticulous individual assessment we organized the patients into two groups; group I consisted of patients undergoing IT gentamicin injection and group II patients undergoing labyrinthectomy, without any preoperative IT gentamicin application. None of the patients had known contralateral ear disease. Patients who would potentially benefit from either intervention were offered both options and were treated with the method of their choice.

Group I included seven patients treated with IT gentamicin application; three patients with unilateral Meniere's disease and four patients with vestibular schwannoma, planned for translab-

\section{H I G G H L I I G}

- Six-canal video-head-impulse test shows that osseous labyrinthectomy causes abnormal contralateral vestibular responses, while chemical ablation of the labyrinthine function with intratympanic gentamicin injections does not.

- Both, surgical labyrinthectomy and chemical ablation with gentamicin result in ipsilateral abnormal or non-recorded vestibular function, as expected.

- Intratympanic gentamicin ablates first the lateral semicircular canal; the superior semicircular canal, possibly because of gravity, is the last one to be affected. yrinthine resection, as part of the prerehabilitation management scheme utilized in our department. Group II consisted of six patients; three patients undergoing a translabyrinthine resection of a vestibular schwannoma, one to obtain clear margins in temporal bone malignancy, one to remove petrous apex cholesteatoma and one for Meniere's disease following failure of IT steroid injections (patient declined any further IT treatment). All patients from both groups underwent six-canal vHIT in predetermined time intervals.

\section{IT gentamicin injections and labyrinthectomy}

All IT injections were performed in the same standardized way by the same surgeon; the number of injections required ranged from two to three with 1-week interval between the injections; enough time to assess the patient clinically, perform vHIT and decide if additional injections were required.

A $40 \mathrm{mg} / \mathrm{mL}$ solution of Gentamicin was utilized with approximately $0.6-0.8 \mathrm{~mL}$ of solution instilled into the middle ear via a $22-G$ spinal needle under topical anaesthesia using the surgical microscope. The patients were asked to keep their head turned towards the contralateral side for 30 minutes after the injection. The labyrinthectomy took place in the same tertiary center, performed in also a well-described standardized way until the complete labyrinth including the three semicircular canals, the vestibule and the "bony island" had been drilled away.

\section{Six-canal vHIT}

Six-canal vHIT were performed by experienced, subspecialized audiologists using the Otometrics six-canal vHIT device and software (Otometrics, Taastrup, Denmark) in a targeted velocity of $>200^{\circ} / \mathrm{sec}$ to ensure accurate, reproducible results. A vestibular-ocular reflex gain between 0.8 and 1.2 was considered normal [9]; we additionally analyzed the raw data, including covert and overt saccades for each semicircular canal to identify abnormal responses.

The patients in group I underwent preoperative vHITs as well as 3 to 5 days following each injection to monitor their response to gentamicin and ensure that pharmacological ablation of the labyrinth had been achieved. We documented the order that each canal responded to gentamicin for every case until all canals had been ablated.

The labyrinthectomy group also had baseline vHIT conforming preoperative normal contralateral responses. Following surgery all patients underwent postoperative six-canal vHIT, 12 to 42 weeks postoperatively at the stage when the patients had no imbalance symptoms and were fit for such testing. All patients from group II were clinically assessed 6 weeks, 12 weeks, 6 months and 12 months following the treatment but vHIT was performed when they were clinically fit and asymptomatic.

\section{Outcome measures}

Our main outcome measures were the contralateral semicircular 
canal responses on vHIT. We documented the vHIT gain for each individual canal, on both the ipsilateral and contralateral side in cases of IT gentamicin injection and on the contralateral side in cases of labyrinthectomy; we also analyzed the raw data looking for covert and/or overt saccades. Additionally we documented demographic factors. We did not include hearing data, mainly because this was not the aim of our study but also because many of the patients were already suffering from severe to profound hearing loss prior to intervention; thus hearing was not tested following IT gentamicin injections. As expected, no measureable hearing was detected ipsilaterally following the labyrinthectomy.

For the patients in group I, we also documented the gain/abnormal response for each canal following every injection in order to determine the precise order that the canals respond to IT gentamicin application. We did not randomize our patients because of the heterogeneity of the groups and because we felt it was not ethical to do so.

\section{RESULTS}

\section{Demographics}

A total of 13 patients, four males and nine females, were enrolled in this study; seven patients underwent IT gentamicin injection and six labyrinthectomy (Table 1 ). The average age for the patients in group I was 45.9 years (range, 27 to 66 years) while for group II 47 years (range, 25 to 70 years).

Table 1. Demographic data

\begin{tabular}{|c|c|c|c|c|c|}
\hline Patient & $\begin{array}{l}\text { Age } \\
(y r)\end{array}$ & Sex & Side & Pathology & Treatment \\
\hline \multicolumn{6}{|c|}{ Group I } \\
\hline 1 & 28 & $\mathrm{~F}$ & $L$ & Vestibular schwannoma & IT gentamicin \\
\hline 2 & 27 & M & $\mathrm{R}$ & Vestibular schwannoma & IT gentamicin \\
\hline 3 & 39 & $\mathrm{~F}$ & $\mathrm{R}$ & Vestibular schwannoma & IT gentamicin \\
\hline 4 & 66 & $\mathrm{~F}$ & $L$ & Vestibular schwannoma & IT gentamicin \\
\hline 5 & 63 & $\mathrm{~F}$ & $\mathrm{~L}$ & Meniere's disease & IT gentamicin \\
\hline 6 & 57 & $\mathrm{~F}$ & $L$ & Meniere's disease & IT gentamicin \\
\hline 7 & 41 & $\mathrm{~F}$ & $\mathrm{R}$ & Meniere's disease & IT gentamicin \\
\hline \multicolumn{6}{|c|}{ Group II } \\
\hline 1 & 38 & $M$ & $L$ & Vestibular schwannoma & $\begin{array}{l}\text { Translabyrinthine } \\
\text { resection }\end{array}$ \\
\hline 2 & 49 & $\mathrm{M}$ & $\mathrm{R}$ & Vestibular schwannoma & $\begin{array}{l}\text { Translabyrinthine } \\
\text { resection }\end{array}$ \\
\hline 3 & 25 & $\mathrm{~F}$ & $L$ & Vestibular schwannoma & $\begin{array}{l}\text { Translabyrinthine } \\
\text { resection }\end{array}$ \\
\hline 4 & 70 & $\mathrm{~F}$ & $\mathrm{R}$ & Meniere's disease & Labyrinthectomy \\
\hline 5 & 55 & $\mathrm{~F}$ & $\mathrm{R}$ & $\begin{array}{l}\text { Temporal bone } \\
\text { carcinoma }\end{array}$ & Total petrosectomy \\
\hline 6 & 45 & $\mathrm{M}$ & $\mathrm{R}$ & $\begin{array}{l}\text { Petrous apex } \\
\text { cholesteatoma }\end{array}$ & $\begin{array}{l}\text { Translabyrinthine } \\
\text { resection }\end{array}$ \\
\hline
\end{tabular}

F, female; M, male; L, left; R, right; IT, intratympanic.

\section{Responses from the ipsilateral side}

From group I, as expected, all patients had abnormal gain and responses in the raw data from the ipsilateral ear, following labyrinthectomy. From group II, in all patients ablation of all three ipsilateral canals was achieved following two to three injections (average 2.4 injections). Following the first IT injection, the lateral semicircular showed abnormal gain in six out of seven ears $(85.7 \%)$, while the posterior semicircular canal in four out of seven $(57 \%)$ and the superior in one out of seven (14.3\%). Following the second injection, the lateral and the posterior canals were ablated in all cases $(100 \%)$, while the superior in five out of seven $(71.4 \%)$. A third injection was required in two patients to ablate the superior semicircular canal. We had no patients with three-canal response to just one injection. Only in one patient the superior canal responded before the posterior; in all cases the lateral canal responded first to gentamicin. In summary, the lateral semicircular canal was the most sensitive to IT gentamicin injections followed by the posterior canal. The superior is usually ablated last (Fig. 1).

\section{Responses from the contralateral ear}

None from the patients from group II had abnormal responses from the contralateral ear, either following each injection or after the complete ablation. In particular, the raw data from the contralateral ears were normal for the patients treated with IT gentamicin injection without any convert or overt saccades (Table 2).

On the other hand, patients in group I undergoing labyrinthectomy had abnormal responses from at least one contralateral semicircular canal, with abnormal gains and coverts/overt saccades (Table 2). Only in one patient, the vHIT gains from the contralateral ear were within normal range; however, analysis of the raw data, showed abnormal responses (covert saccades) from the contralateral lateral semicircular canal.

\section{DISCUSSION}

IT gentamicin injections and labyrinthectomy have both been used over the years to treat disabling, unilateral vestibular disease, while the labyrinthectomy has also been used to access and remove temporal bone/skull base pathology. The impact of such interventions on the affected ear is definite and irreversible [1-4]. However, their effect on the contralateral, healthy ear has not been investigated, until now. We present, to our knowledge for the first time, abnormal, repeatable responses recorded from the contralateral ear in all enrolled individuals undergoing labyrinthectomy; on the other hand, none of the patients treated with IT gentamicin application had any contralateral abnormal responses. We additionally, demonstrated in vivo, the order that the semicircular canals respond to IT gentamicin injections, with the lateral one being the most sensitive and the superior one the most resistant. 

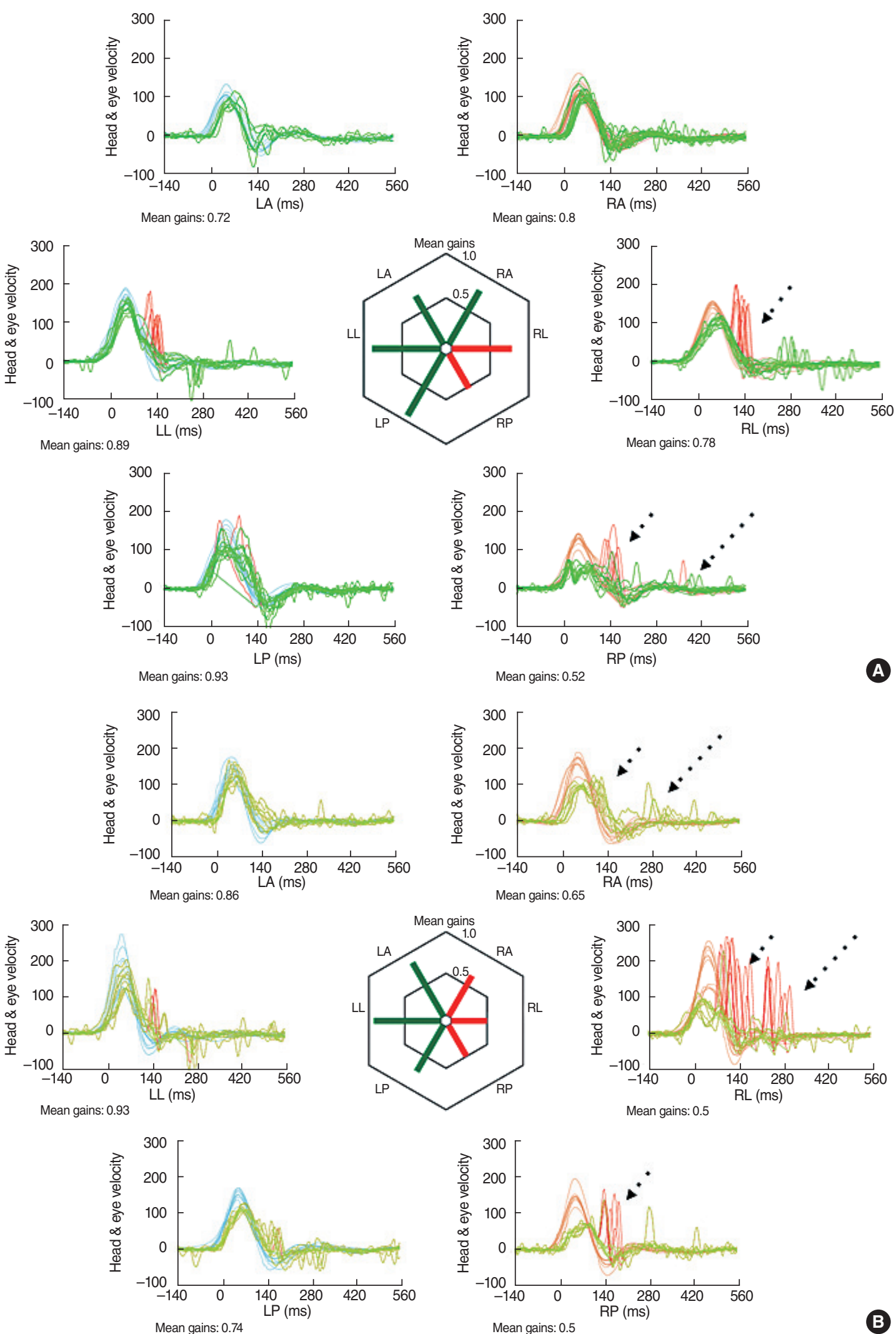

A 
Table 2. Responses from the contralateral labyrinth

\begin{tabular}{|c|c|c|c|c|}
\hline Patient & $\begin{array}{l}\text { Gain ctl } \\
\text { LSCC }\end{array}$ & $\begin{array}{l}\text { Gain ctl } \\
\text { PSCC }\end{array}$ & $\begin{array}{l}\text { Gain ctl } \\
\text { SSCC }\end{array}$ & Raw data abnormality \\
\hline \multicolumn{5}{|l|}{ Group I } \\
\hline 1 & 0.98 & 0.85 & 1.07 & None \\
\hline 2 & 0.79 & 0.87 & 0.87 & None \\
\hline 3 & 0.95 & 0.83 & 0.89 & None \\
\hline 4 & 0.76 & 1.08 & 0.70 & Normal raw data despite $<0.8$ gain \\
\hline 5 & 0.85 & 0.95 & 1.13 & None \\
\hline 6 & 0.91 & 0.82 & 0.83 & None \\
\hline 7 & 0.85 & 0.86 & 1.09 & None \\
\hline \multicolumn{5}{|c|}{ Group II } \\
\hline 1 & $0.69^{a)}$ & $0.63^{\mathrm{a})}$ & $0.6^{\mathrm{a})}$ & $\begin{array}{l}\text { Covert/overt saccades from all three } \\
\text { canals }\end{array}$ \\
\hline 2 & $0.68^{\text {a) }}$ & 0.78 & 1.04 & Covert/overt saccades from LSCC \\
\hline 3 & $1.15^{\mathrm{a})}$ & 1 & 1.04 & Covert/overt saccades from LSCC \\
\hline 4 & 0.9 & $0.77^{\mathrm{a})}$ & $0.9^{\mathrm{a})}$ & Covert saccades from LSCC \\
\hline 5 & 0.77 & $0.6^{\mathrm{a})}$ & $0.48^{\mathrm{a})}$ & $\begin{array}{l}\text { Covert/overt saccades from the } \\
\text { PSCC and SSCC }\end{array}$ \\
\hline 6 & 1.15 & $0.79^{\text {a) }}$ & $0.99^{a)}$ & Covert saccades from PSCC \\
\hline
\end{tabular}

The gain documented refers to the gain following the final measurement; however, no abnormal responses were documented from the contralateral ear in group I at any stage.

ctl, contralateral; LSCC, lateral semicircular canal; PSCC, posterior semicircular canal; SSCC, superior semicircular canal.

a) Values indicate definite abnormal responses based on the raw data interpretation.

\section{The significance of contralateral responses}

To date, the impact of unilateral vestibular deafferentation on the contralateral labyrinth and the central system has been poorly examined and understood. An older study showed reduced vestibular-ocular reflex gain in the contralateral ear immediately after labyrinthectomy in monkeys, with later recovery [11]. Additionally, recent work investigating contralateral vHIT responses following retrosigmoidal vestibular schwannoma resection showed the same short-term reduction in gains followed by gradual stabilization [12], while previous work reported no long-term recovery at all, in certain patients [13]. Our study shows similar results following surgical labyrinthectomy with abnormal contralateral responses several months following the initial intervention. We did not perform any immediate measurements like Mantokoudis et al. [12] because of the practical challenge of performing six-canal vHIT following major surgery; therefore we were unable to assess the immediate postoperative function; however, the abnormal gain was evident in all patients following labyrinthectomy in at least one canal many months down the line. Of note that in our study we tested all six canals and not only the lateral one.

One could assume that in the three patients who underwent labyrinthectomy for a vestibular schwannoma resection, additional vestibular nerve sacrifice was performed to remove the tumor with such procedure adding to the vestibular trauma. However, the same results were recorded in the other three pa- tients who did not have any intervention on the vestibular nerve. Thus, the responses generated by the contralateral ear are more likely the result of the labyrinthectomy rather than any additional manipulations.

The reason behind such abnormal responses is difficult to prove. However, a process of on going central compensation is a likely plausible explanation. Indeed, the previously reported hypothesis of inhibited contralateral vestibular pathway by the central nervous system as part of central compensation following acute vestibular damage is a possible explanation [12,14].

On the other hand, we did not identify any contralateral gain changes following IT gentamicin injections, despite the ipsilateral reduced gains documented in all three canals. Although the precise, underlying mechanism of such finding is not entirely clear, we hypothesize that it might reflect the gradual impact of gentamicin on the labyrinth and the central compensation mechanisms, in contrast to the sudden and rapid damage achieved through surgery. Interestingly, the vHITs were performed much later in the labyrinthectomy group, which gave the patient more time to compensate. Still, though, these patients demonstrated abnormal contralateral responses, while the patients from the gentamicin group, tested only five days post-injection (in theory less time to compensate), did not produce any contralateral abnormal responses.

Another possible explanation could be that the reduced ipsilateral gains following IT gentamicin application do not necessarily mean that there is no remaining ipsilateral vestibular function, while in case of labyrinthectomy there is no remaining function at all. Such "less traumatic" impact of IT gentamicin is still able to improve the patients' symptoms by reducing the ipsilateral vestibular function rather than completely ablating it; consequently it generates milder contralateral responses. Indeed, the presence of covert and/or overt saccades is part of the compensation mechanism as a response to unilateral vestibular deafferentation $[12,15]$. The lack of such contralateral saccades following IT injections but their presence after labyrinthectomy indicate a milder and possibly more gradual impact of gentamicin compared to the rapid and sudden damage caused surgically.

Interestingly, Roehm et al. [16] reported traces of gentamicin in the contralateral ear in chinchillas following IT gentamicin injections. The authors attributed this transfer of gentamicin from one side to the other to the cochlear aqueduct [16]. Our study did not examine traces of gentamicin on micro-level; only vHIT responses from the contralateral ear, which in the gentamicin group were all normal. Whether there is transfer of gentamicin to the contralateral ear via the cochlear aqueduct in the human ear, it is unknown. The normal contralateral responses shown in our study suggest that even if such transfer occurs in human, it is probably not sufficient enough to cause abnormal vHIT results.

To our knowledge, there is a lack of similar studies in the literature; our work offers the first direct comparison of labyrinthectomy and IT gentamicin injections. Additional studies will 
be of benefit for shedding more light on the indirect impact of radical interventions on the contralesional side and the central compensation mechanisms.

\section{Semicircular canal responses to IT gentamicin}

We have shown a relative resistance of the superior canal to IT gentamicin injection, while the lateral canal was the first one to show abnormal gain, followed by the posterior. Such finding is probably related to the anatomy. Indeed, following IT injection, the gentamicin enters the inner ear through the round and oval windows. Given the horizontal, slightly elevated position of the patient's head, the lateral semicircular canal is the first one to be affected. On the other hand, because of gravity the superior canal can be expected to respond last.

Marques et al. [17] found reduced ipsilateral gain in all canals following one IT gentamicin injection in some patients. As shown by previous study looking into the ototoxicity of systemic gentamicin, such agent can be vestibulotoxic in any dose and in any regime [8]. Thus, there should be patients who will respond to even one IT gentamicin injection without the need for a repeat application. We did not record similar responses, as we had no case with abnormal responses in all three ipsilateral canals following just one injection. This might be either because of the enrolled numbers or even related to the method of IT application and the presence of middle ear related factors such as adhesions. However, the ipsilateral vestibular-ocular gains finally reduced in all canals following a maximum of three injections.

\section{Strengths and limitations}

The novelty and the prospective structure of our work are its main strengths. This is the first study directly comparing the impact of IT gentamicin application with the impact of labyrinthectomy on the contralateral side. At the same time, using six-canal vHIT we showed the order that the canals respond to IT gentamicin injections. Utilizing the six-canal vHIT technique allowed us to assess all canals in an accurate way, as it has been proven as a quick and repeatable way of assessing all six semicircular canals $[8,12,18,19]$.

The main limitation of the study is the relatively small number of the enrolled patients. Additionally, we did not randomize our patients because of the heterogeneity of the groups and ethical considerations when offering radical interventions. However, this is a pilot study involving radical unilateral vestibular ablation as part of an on going time-demanding project on vestibular assessment; thus we did not include audiological outcomes (radical treatments, profound/complete hearing loss). The tertiary settings that allowed us to recruit individuals and also to perform the interventions and the repeated six-canal vHITs in a controlled environment ensure accurate results and careful data interpretation, helping us to overcome the limitation of small numbers.

Our study shows abnormal responses from the contralateral ear following labyrinthectomy even many months following the treatment. On the other hand, IT gentamicin does not generate such responses while at the same time it achieves the desired ipsilateral reduced vestibular response, making it a radical treatment of choice compared to labyrinthectomy for unilateral vestibular dysfunction. Finally, IT gentamicin seems to affect the ipsilateral canals in a certain order with the lateral semicircular canal being the first one to be ablated.

\section{CONFLICT OF INTEREST}

No potential conflict of interest relevant to this article was reported.

\section{ACKNOWLEDGMENTS}

This work was in part presented at the Otology section, Royal Society of Medicine, February 2, 2018, London, UK.

\section{ORCID}

Georgios Kontorinis https://orcid.org/0000-0002-1637-1661

\section{AUTHOR CONTRIBUTIONS}

Conceptualization: GK, MG. Data curation: GK, HT, MG. Formal analysis: GK, HT, JAC. Funding acquisition: GK. Methodology: GK, JAC, HT. Project administration: HT, MG. Visualization: HT, GK. Writing - original draft: HT, GK. Writing - review \& editing: HT, JAC, GK.

\section{REFERENCES}

1. Pullens B, van Benthem PP. Intratympanic gentamicin for Meniere's disease or syndrome. Cochrane Database Syst Rev. 2011 Mar;(3): CD008234.

2. Sajjadi H, Paparella MM. Meniere's disease. Lancet. 2008 Aug;372 (9636):406-14.

3. Rah YC, Han JJ, Park J, Choi BY, Koo JW. Management of intractable Meniere's disease after intratympanic injection of gentamicin. Laryngoscope. 2015 Apr;125(4):972-8.

4. Ghossaini SN,Wazen JJ.An update on the surgical treatment of Meniere's diseases. J Am Acad Audiol. 2006 Jan;17(1):38-44.

5. Buki B, Junger H. Intratympanal gentamicin in Meniere's disease: effects on individual semicircular canals. Auris Nasus Larynx. 2018 Feb;45(1):39-44.

6. Tjernstrom F, Fransson PA, Kahlon B, Karlberg M, Lindberg S, Siesjo $\mathrm{P}$, et al. Hearing and vestibular function after preoperative intratympanic gentamicin therapy for vestibular schwanomma as part of vestibular prehab. Ear Hear. 2016 Nov/Dec;37(6):744-50. 
7. Marques PS, Dias CC, Perez-Fernandez N, Spratley J. Instrumental head impulse test changes after intratympanic gentamicin for unilateral definite Meniere's disease: a systematic review and meta-analysis. Auris Nasus Larynx. 2018 Oct;45(5):943-51.

8. Ahmed RM, Hannigan IP, MacDougall HG, Chan RC, Halmagyi GM. Gentamicin ototoxicity: a 23-year selected case series of 103 patients. Med J Aust. 2012 Jun;196(11):701-4.

9. Weber KP, Aw ST, Todd MJ, McGarvie LA, Curthoys IS, Halmagyi GM. Horizontal head impulse test detects gentamicin vestibulotoxicity. Neurology. 2009 Apr;72(16):1417-24.

10. MacDougall HG, Weber KP, McGarvie LA, Halmagyi GM, Curthoys IS. The video head impulse test: diagnostic accuracy in peripheral vestibulopathy. Neurology. 2009 Oct;73(14):1134-41.

11. Fetter M, Zee DS. Recovery from unilateral labyrinthectomy in rhesus monkey. J Neurophysiol. 1988 Feb;59(2):370-93.

12. Mantokoudis G, Schubert MC, Tehrani AS, Wong AL, Agrawal Y. Early adaptation and compensation of clinical vestibular responses after unilateral vestibular deafferentation surgery. Otol Neurotol. 2014 Jan;35(1):148-54.
13. Tian JR, Ishiyama A, Demer JL. Temporal dynamics of semicircular canal and otolith function following acute unilateral vestibular deafferentation in humans. Exp Brain Res. 2007 Apr;178(4):529-41.

14. McCabe BF, Ryu JH, Sekitani T. Further experiments on vestibular compensation. Laryngoscope. 1972 Mar;82(3):381-96.

15. Blodow A, Pannasch S, Walther LE. Detection of isolated covert saccades with the video head impulse test in peripheral vestibular disorders. Auris Nasus Larynx. 2013 Aug;40(4):348-51.

16. Roehm P, Hoffer M, Balaban CD. Gentamicin uptake in the chinchilla inner ear. Hear Res. 2007 Aug;230(1-2):43-52.

17. Marques P, Manrique-Huarte R, Perez-Fernandez N. Single intratympanic gentamicin injection in Meniere's disease: VOR change and prognostic usefulness. Laryngoscope. 2015 Aug;125(8):1915-20.

18. Stevens MN, Garrison DB, Kaylie DM. What is the potential clinical utility of vHIT when assessing adult patients with dizziness? Laryngoscope. 2017 Dec;127(12):2689-90.

19. Batuecas-Caletrio A, Santa Cruz-Ruiz S, Munoz-Herrera A, PerezFernandez N. The map of dizziness in vestibular schwannoma. Laryngoscope. 2015 Dec;125(12):2784-9. 